\title{
SYSTEM PERFORMANCES OF SC RECEPTION IN ASYMMETRIC MULTIPATH FADING ENVIRONMENTS
}

\author{
DANIJEL ĐOŠIĆ ${ }^{1 *}$, ČASLAV STEFANOVIĆ ${ }^{1}$, DEJAN MILIĆ ${ }^{2}$, MIHAJLO STEFANOVIĆ
}

${ }^{1}$ Faculty of Natural Science and Mathematics, University of Priština, Kosovska Mitrovica, Serbia

${ }^{2}$ Faculty of Electronic Engineering, University of Niš, Niš, Serbia

\begin{abstract}
The paper takes into consideration dual-branch selection combining (SC) diversity reception technique in the presence of dissimilar fading at their branches. We efficiently obtained first and second order closed form statistics at the output of the considered model such as probability density function (PDF), cumulative distribution function (CDF) and average level crossing rate (aLCR). Moreover, obtained results a re numerically presented for various sets of system model parameters and mainly confirmed by computer based MATLAB simulations.
\end{abstract}

Keywords: Selection combining (SC), Nakagami- $m$ random variable, $\alpha-\mu$ random variable, PDF, CDF, aLCR.

\section{INTRODUCTION}

Fast fading (multipath) has a large impact on the error probability of a wireless telecommunications systems, as well as on the channel information capacity. What kind of distribution will be used to describe the wireless channel in the presence of the fading depends on: i.) whether there is a dominant component, ii.) whether the conditions at the reception have been met that satisfy a central limit theorem, iii.) whether environment has one or multiple clusters and etc. The most commonly used distributions are Rayleigh, Rice, Nakagami- $m, \eta-\mu, \alpha-\mu$, and $\kappa-\mu$ (Simon \& Alouini, 2000; Panic et al., 2017; Nakagami, 1960; Yacoub, 2007; Djosic et al., 2016; Stefanović \& Đošić, 2016). Various diversity techniques are used to reduce the impact of the fast fading on the system performances. Diversity techniques, due to their ability to minimize the effects of fading, are one of the most adequate ways to improve the reliability of the transmission without increasing transmission power and bandwidth. There are several types of diversity combining techniques whose division can in general be made depending on the constraints regarding the complexity of the communication systems and the availability of channel status information (CSI) at the receiver (Simon \& Alouini, 2000; Panic et al., 2017).

Selection combining (SC) receiver is one of the simplest and mainly used diversity combining techniques. The SC receiver may have two, three or more inputs. In the case where the $\mathrm{SC}$ receiver has two inputs, the signal at the output is equal to the stronger signal at their inputs. Hardware solution of a commercial SC combiner with two inputs is shown in Fig. 1. (Analog Devices, 2015).

Regardless of the fact that other diversity techniques can provide better results (for example, maximal ratio combining and equal gain combining) (Simon \& Alouini, 2000; Panic et al., 2017), SC technique due to its simplicity has important

* Corresponding author: danijel.djosic@pr.ac.rs

MATHEMATICS, COMPUTER SCIENCE AND MECHANICS application in various wireless communications systems. The papers (Milic et al., 2016; Stefanović et al., 2013; Panajotović et al., 2013) consider first and second order performance analysis of dual branch SC receiver in the presence of co-channel interference. In paper, referenced as (Stefanović \& Đošić, 2016), the macro-diversity system is considered with SC reception at micro-level. Moreover, the paper (Djosic et al., 2016; Stefanovic et al., 2017) consider second order statistics of macro-diversity systems with SC at micro-level as well as at macro-level. The vehicle-to-vehicle (V2V) cooperative relay wireless communications over fading channels with SC are considered in (Stefanović et al., 2018; Bithas et al., 2016) while high speed train (HST) wireless communications with selection techniques are investigated in (Laiyemo et al., 2017). Some further insights in selection scheme strategies for wireless transmission are provided in (Swaminathan et al., 2016; Y1lmaz \& Kucur, 2014; Stefanovic, 2017).

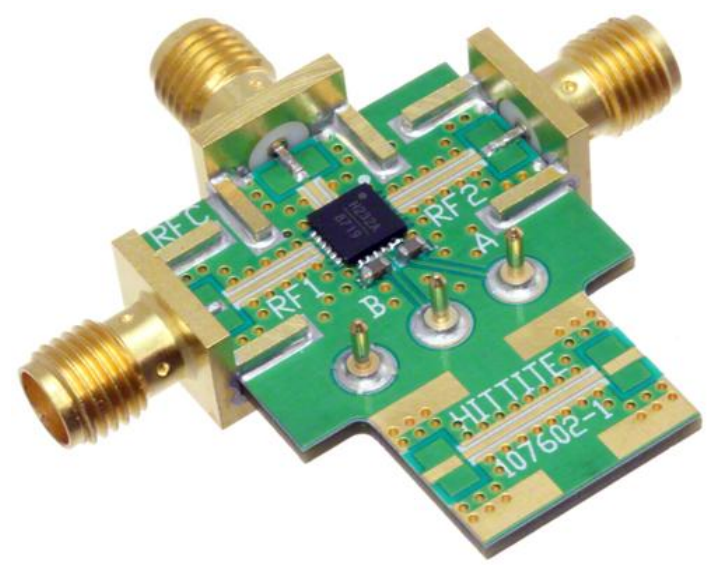

Figure 1. Evaluation board of a commercial dual-branch SC combiner hardware implementation.

This paper analyses SC receiver with two inputs in the presence of Nakagami- $m$ fading on one branch and $\alpha-\mu$ fading on the other branch. Probability density function (PDF), cumulative 
density function (CDF), moments, joint probability density function and level crossing rate (aLCR) of the proposed SC system are calculated, as the closed form expressions and numerically evaluated for various system model set of parameters. Moreover, some obtained results are confirmed by MATLAB simulations.

\section{SYSTEM MODEL}

In this section, we consider SC receiver with two inputs. At the first input Nakagami- $m$ multipath fading is presented while at the second input $\alpha-\mu$ fading is presented. The block scheme system model is presented in Fig. 2.

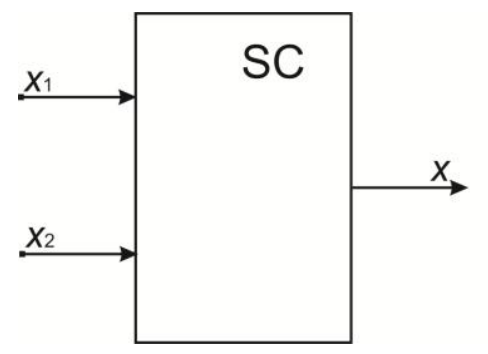

Figure 2. SC block scheme reception with two inputs.

Signals at the inputs are denoted with $x_{1}$ and $x_{2}$. The output signal is denoted with $x$. The Nakagami- $m$ distribution describes the signal envelope in linear environments (Nakagami, 1960), while the $\alpha-\mu$ distribution describes signal envelope in environment whose dispersion field is not homogeneous (Yacoub, 2007). The probability density function of Nakagami- $m$ random variable $x_{1}$ is (Simon \& Alouini, 2000; Panic et al., 2017; Nakagami, 1960):

$$
p_{x_{1}}\left(x_{1}\right)=\frac{2}{\Gamma\left(m_{1}\right)}\left(\frac{m_{1}}{\Omega_{1}}\right)^{m_{1}} x_{1}^{2 m_{1}-1} e^{-\frac{m_{1}}{\Omega_{1}} x_{1}^{2}}, \quad x_{1} \geq 0 .
$$

where $m_{1}$ is fading severity parameter, $\Omega_{1}$ is mean power. The cumulative distribution function of $x_{1}$ is obtained by integration of Nakagami- $m$ probability density function (Simon \& Alouini, 2000; Panic et al., 2017; Nakagami, 1960):

$$
F_{x_{1}}\left(x_{1}\right)=\frac{1}{\Gamma\left(m_{1}\right)} \gamma\left(m_{1}, \frac{m_{1}}{\Omega_{1}} x_{1}^{2}\right), \quad x_{1} \geq 0 .
$$

The $n$-th order moment of $x_{1}$ or the mean $n$-th value is equal to (Gradshteyn \& Ryzhik, 2000):

$$
\begin{aligned}
m_{n x_{1}} & =\overline{x_{1}^{n}}=\int_{0}^{\infty} d x_{1} x_{1} p_{x_{1}}\left(x_{1}\right)=\frac{2}{\Gamma\left(m_{1}\right)}\left(\frac{m_{1}}{\Omega_{1}}\right)^{m_{1}} \\
& \times \int_{0}^{\infty} d x_{1} x_{1}^{2 m_{1}-1+n} e^{-\frac{m_{1}}{\Omega_{1}} x_{1}^{2}}=\frac{1}{\Gamma\left(m_{1}\right)}\left(\frac{\Omega_{1}}{m_{1}}\right)^{n / 2} \Gamma\left(m_{1}+\frac{n}{2}\right) .
\end{aligned}
$$

The first and second moments of Nakagami- $m$ random variable are:

$$
\begin{gathered}
m_{1}=\frac{1}{\Gamma\left(m_{1}\right)}\left(\frac{\Omega_{1}}{m_{1}}\right)^{1 / 2} \Gamma\left(m_{1}+\frac{1}{2}\right) . \\
m_{2}=\overline{x_{1}^{2}}=\frac{1}{\Gamma\left(m_{1}\right)} \frac{\Omega_{1}}{m_{1}} \Gamma\left(m_{1}+1\right)=\frac{1}{\Gamma\left(m_{1}\right)} \frac{\Omega_{1}}{m_{1}} m_{1} \Gamma\left(m_{1}\right)=\Omega_{1} .
\end{gathered}
$$

where $\Omega_{1}$ is by definition the mean square value. The first moment is the mean value, and the second moment is the mean square value. The third-order moment is equal to:

$$
m_{3}=\frac{1}{\Gamma\left(m_{1}\right)}\left(\frac{\Omega_{1}}{m_{1}}\right)^{3 / 2} \Gamma\left(m_{1}+\frac{3}{2}\right) .
$$

Relations can be formed between the usual moments and central moments.

The variance is equal to the difference between the mean square value and the mean value per square. The variation of $x_{1}$ is:

$$
\sigma_{x_{1}}^{2}=\overline{x_{1}^{2}}-\bar{x}_{1}^{2}
$$

The joint probability density function from Nakagami- $m$ random process and its first derivative can be obtained as follows. The square of Nakagami- $m$ random process is equal to the sum of $2 m$ square Gauss random variables with zero mean values and with the same variations. The variable $x_{2}$ has $\chi^{2}$ distribution (Simon \& Alouini, 2000; Panic et al., 2017; Nakagami, 1960). Based on this, $x$ has a Nakagami- $m$ distribution:

$$
x^{2}=x_{1}^{2}+x_{2}^{2}+\ldots+x_{2 m}^{2}
$$

The first derivative of $x$ is:

$$
\dot{x}=\frac{1}{x}\left(x_{1} \dot{x}_{1}+x_{2} \dot{x}_{2}+\ldots+x_{2 m} \dot{x}_{2 m}\right) .
$$

The first derivative of Gaussian random variable is Gaussian random variable. A linear combination of Gaussian random variables is Gaussian random variable. On this basis the first derivative of $x$ has Gaussian distribution (Middleton, 1960). The mean value is:

$$
\overline{\dot{x}}=\frac{1}{x}\left(x_{1} \overline{\dot{x}}_{1}+x_{2} \overline{\dot{x}}_{2}+\ldots+x_{2 m} \overline{\dot{x}_{2 m}}\right)=0 .
$$

The mean values of Gaussian first derivatives are equal to zero: $\overline{\dot{x}_{1}}=\overline{\dot{x}_{2}}=\ldots=\overline{\dot{x}_{2 m}}=0$.

The variance of $\dot{x}$ is obtained as the variance summation of each member. The variance of $\dot{x}$ is equal to the product of the square of the constant and the square of the variance of the random variable. Based on this:

$$
\sigma_{\dot{x}}^{2}=\frac{1}{x^{2}}\left(x_{1}^{2} \sigma_{\dot{x}_{1}}^{2}+x_{2}^{2} \sigma_{\dot{x}_{2}}^{2}+\ldots+x_{2 m}^{2} \sigma_{\dot{x}_{2 m}}^{2}\right) .
$$


where, $\sigma_{\dot{x}_{1}}^{2}=\sigma_{\dot{x}_{2}}^{2}=\ldots=\sigma_{\dot{x}_{2 m}}^{2}=2 \pi^{2} f_{m}^{2} \sigma^{2}=\pi^{2} f_{m}^{2} \Omega_{1} / m_{1}$.

The variance of the first derivative is equal to the product of $\pi^{2} f_{m}^{2}$ a and standard deviation $\sigma^{2}$, the standard deviation is equal to the ratio, $\Omega_{1} / m_{1}$. By substitution:

$$
\begin{aligned}
\sigma_{\dot{x}}^{2} & =\frac{1}{x^{2}} 2 \pi^{2} f_{m}^{2} \sigma^{2}\left(x_{1}^{2}+x_{2}^{2}+\ldots+x_{2 m}^{2}\right)= \\
& =\frac{1}{x^{2}} 2 \pi^{2} f_{m}^{2} \sigma^{2} x^{2}=2 \pi^{2} f_{m}^{2} \sigma^{2} .
\end{aligned}
$$

The probability density function of $\dot{x}$ is Gaussian, since the linear combination of Gaussian random variables is a Gaussian (Middleton, 1960):

$$
p_{\dot{x}}(\dot{x})=\frac{1}{\sqrt{2 \pi} \sigma_{\dot{x}}} e^{-\frac{\dot{x}^{2}}{\sigma_{\dot{x}}^{2}}}
$$

where $\sigma_{\dot{x}}^{2}$ is a variance of Gaussian $\dot{x}$ distribution.

Random variables $x$ and $\dot{x}$ are mutually independent. This can be proved numerically. Based on this, joint probability density function of $x$ and $\dot{x}$ is equal to the product of a Nakagami- $m$ random variable and a Gaussian random variable.

$$
p_{x_{1} \dot{x}_{1}}\left(x_{1} \dot{x}_{1}\right)=\frac{2}{\Gamma\left(m_{1}\right)}\left(\frac{m_{1}}{\Omega_{1}}\right)^{m_{1}} x_{1}^{2 m_{1}-1} e^{-\frac{m_{1}}{\Omega_{1}} x_{1}^{2}} \frac{1}{\sqrt{2 \pi} \sigma_{\dot{x}_{1}}^{2}} e^{-\frac{\dot{x}_{1}^{2}}{2 \sigma_{\dot{x}_{1}}^{2}}}
$$

To determine the average level crossing rate (aLCR) of the random process, the joint probability density function of the random variable and the first derivative of the random variable should be determined. The aLCR of $x_{1}$ can be calculated as mean of the random process (Simon \& Alouini, 2000; Panic et al., 2017):

$$
N_{x_{1}}=\int_{0}^{\infty} d \dot{x}_{1} \dot{x}_{1} p_{x_{1} \dot{x}_{1}}\left(x_{1} \dot{x}_{1}\right)=\frac{2}{\Gamma\left(m_{1}\right)}\left(\frac{m_{1}}{\Omega_{1}}\right)^{m_{1}} x_{1}^{2 m_{1}-1} e^{-\frac{m_{1}}{\Omega_{1}} x_{1}^{2}} \frac{\sigma_{\dot{x}_{1}}}{\sqrt{2 \pi}} .
$$

where: $\sigma_{\dot{x}_{1}}^{2}=\pi^{2} f_{m}^{2} \Omega_{1} / m_{1}$.

The random variable $x_{2}$ follow an $\alpha-\mu$ distribution. The distribution describes signal in a nonlinear and nonhomogeneous environment (Simon \& Alouini, 2000; Panic et al., 2017; Yacoub, 2007)

$$
p_{x_{2}}\left(x_{2}\right)=\frac{\alpha}{\Gamma\left(m_{2}\right)}\left(\frac{m_{2}}{\Omega_{2}}\right)^{m_{2}} x_{2}^{\alpha m_{2}-1} e^{-\frac{m_{2}}{\Omega_{2}} x_{2}^{2}}, \quad x_{2} \geq 0 .
$$

The relation between $x_{1}$ and $x_{2}$ is:

$$
x_{2}=x_{1}^{2 / \alpha}, \quad x_{1}=x_{2}^{2 / \alpha} .
$$

In this case, $x_{1}$ follows a Nakagami- $m$ distribution, and $x_{2}$ follows an $\alpha-\mu$ distribution. The following relation is between Nakagami- $m$ and $\alpha-\mu$ distribution, $m=\mu$. The cumulative distribution probability of $x_{2}$ is obtained by integrating the
Nakagami-m distribution (Simon \& Alouini, 2000; Nakagami, 1960):

$$
\begin{aligned}
F_{x_{2}}\left(x_{2}\right) & =\int_{0}^{x_{2}} d t p_{x_{2}}(t)=\frac{\alpha}{\Gamma\left(m_{2}\right)}\left(\frac{m_{2}}{\Omega_{2}}\right)^{m_{2}} \int_{0}^{x_{2}} d t t^{\alpha m_{2}-1} e^{-\frac{m_{2}}{\Omega_{2}} t^{\alpha}}= \\
& =\frac{1}{\Gamma\left(m_{2}\right)} \gamma\left(m_{2}, \frac{m_{2}}{\Omega_{2}} x_{2}^{\alpha}\right), \quad x_{2} \geq 0 .
\end{aligned}
$$

The $n$-th order moment of $x_{2}$ is (Simon \& Alouini, 2000):

$$
\begin{aligned}
m_{n x_{2}} & =\overline{x_{2}^{n}}=\int_{0}^{\infty} d x_{2} x_{2} p_{x_{2}}\left(x_{2}\right)= \\
& =\frac{\alpha}{\Gamma\left(m_{2}\right)}\left(\frac{m_{2}}{\Omega_{2}}\right)^{m_{2}} \int_{0}^{\infty} d x_{2} x_{2}^{\alpha m_{2}-1+n} e^{-\frac{m_{2}}{\Omega_{2}} x_{2}^{2}}= \\
& =\frac{\alpha}{\Gamma\left(m_{2}\right)}\left(\frac{\Omega_{2}}{m_{2}}\right)^{m_{2}+n / \alpha} \Gamma\left(m_{2}+\frac{n}{\alpha}\right) .
\end{aligned}
$$

The mean value for the $\alpha-\mu$ random variate is obtained for $n=1$ :

$$
m_{x_{21}}=\frac{1}{\alpha}\left(\frac{\Omega_{2}}{m_{2}}\right)^{m_{2}+1 / 2} \Gamma\left(m_{2}+\frac{1}{\alpha}\right)
$$

The mean squared value for the $\alpha-\mu$ random variate is obtained for $n=2$ :

$$
m_{22}=\frac{1}{\Gamma\left(m_{2}\right)}\left(\frac{\Omega_{2}}{m_{2}}\right)^{m_{2}+\frac{2}{\alpha}} \Gamma\left(m_{1}+\frac{2}{\alpha}\right) .
$$

The joint probability density function of $\alpha-\mu$ random variable and the first derivative of $\alpha-\mu$ random variable is obtained by following transformations (Panic et al., 2017; Yacoub, 2007):

$$
x_{2}=x_{1}^{2 / \alpha}, \quad x_{1}=x_{2}^{\alpha / 2}, \quad \dot{x}_{2}=\frac{2}{\alpha} x_{1}^{2 / \alpha-1} \dot{x}_{2}, \quad \dot{x}_{1}=\frac{\alpha}{2} x_{2}^{\alpha / 2-1} \dot{x}_{1} .
$$

The joint probability density function of $\alpha-\mu$ random variable and the first derivative of $\alpha-\mu$ random variable can be used to calculate the aLCR of $\alpha-\mu$ random process. The joint probability density function of $x_{2}$ is:

$$
p_{x_{2} \dot{x}_{2}}\left(x_{2} \dot{x}_{2}\right)=|J| p_{x_{1} \dot{x}_{1}}\left(x_{2}^{\alpha / 2}, \frac{\alpha}{2} x_{2}^{\alpha / 2-1} \dot{x}_{2}\right) \text {. }
$$

where $J$ is the Jacobian of the transformation, given as:

$$
J=\left|\begin{array}{ll}
\frac{\partial x_{1}}{\partial x_{2}} & \frac{\partial x_{1}}{\partial \dot{x}_{2}} \\
\frac{\partial \dot{x}_{1}}{\partial x_{2}} & \frac{\partial \dot{x}_{1}}{\partial \dot{x}_{2}}
\end{array}\right|=\left|\begin{array}{cc}
\frac{\alpha}{2} x_{2}^{\alpha / 2-1} & 0 \\
0 & \frac{\alpha}{2} x_{2}^{\alpha / 2-1}
\end{array}\right|=\frac{\alpha^{2}}{4} x_{2}^{\alpha-2}
$$

By substitution, the combined probability density function of the $\alpha-\mu$ random variable and the first derivative of the $\alpha-\mu$ random variable is obtained: 


$$
\begin{gathered}
p_{x_{2} \dot{x}_{2}}\left(x_{2} \dot{x}_{2}\right)=\frac{\alpha^{2}}{4} x_{2}^{\alpha-2} p_{x_{1} \dot{x}_{1}}\left(x_{2}^{\alpha / 2}, \frac{\alpha}{2} x_{2}^{\alpha / 2-1} \dot{x}_{2}\right)=\frac{\alpha^{2}}{4} x_{2}^{\alpha-2} \\
\times \frac{2}{\Gamma\left(m_{1}\right)}\left(\frac{m_{1}}{\Omega_{1}}\right)^{m_{1}} x_{2}^{\frac{\alpha}{2}\left(2 m_{1}-1\right)} e^{-\frac{m_{1}}{\Omega_{1}} x_{2}^{\alpha}} \frac{1}{\sqrt{2 \pi} \sigma_{\dot{x}_{1}}} e^{-\frac{1}{2 \sigma_{\dot{x}_{1}}^{2}} \frac{\alpha^{2}}{4} x_{2}^{\alpha-2} \dot{x}_{2}^{2}} \\
=\frac{\alpha^{2}}{2 \Gamma\left(m_{1}\right)}\left(\frac{m_{1}}{\Omega_{1}}\right)^{m_{1}} x_{2}^{\alpha m_{1}+\frac{\alpha}{2}+2} \frac{1}{\sqrt{2 \pi} \sigma_{\dot{x}_{1}}} e^{-\frac{m_{1}}{\Omega_{1}} x_{2}^{\alpha}} e^{-\frac{\alpha^{2}}{4 \sigma_{\dot{x}_{1}}^{2}} x_{2}^{\alpha-\dot{x}_{2}}} .
\end{gathered}
$$

The aLCR of $x_{2}$ is obtained as the mean of the first derivative of the $\alpha-\mu$ random variable $x_{2}$. The aLCR of the signal at the second input of the SC receiver is (Simon \& Alouini, 2000; Panic et al., 2017):

$$
\begin{aligned}
N_{x_{2}} & =\int_{0}^{\infty} d \dot{x}_{2} \dot{x}_{2} p_{x_{2} \dot{x}_{2}}\left(x_{2} \dot{x}_{2}\right)=\frac{\alpha^{2}}{2 \Gamma\left(m_{1}\right)}\left(\frac{m_{1}}{\Omega_{1}}\right)^{m_{1}} \\
& \times x_{2}^{\alpha m_{1}+\frac{\alpha}{2}+2} e^{-\frac{m_{1}}{\Omega_{1}} x_{2}^{\alpha}} \int_{0}^{\infty} d \dot{x}_{2} \dot{x}_{2} e^{-\frac{\alpha^{2}}{4 \sigma_{x_{1}}} x_{2}^{\alpha-2} \dot{x}_{2}^{2}} \frac{1}{\sqrt{2 \pi} \sigma_{\dot{x}_{2}}}= \\
& =\frac{1}{2 \sqrt{2 \pi} \sigma_{\dot{x}_{2}} \Gamma\left(m_{1}\right)}\left(\frac{m_{1}}{\Omega_{1}}\right)^{m_{1}} x_{2}^{\alpha m_{1}+\frac{\alpha}{2}+2} e^{-\frac{m_{1}}{\Omega_{1}} x_{2}^{\alpha}} \frac{1}{2} \frac{4 \sigma_{\dot{\alpha}_{1}}^{2}}{x_{2}^{\alpha-2}} .
\end{aligned}
$$

The signal probability density function at the output from the $\mathrm{SC}$ receiver is obtained as follows. Signal at the output of the $\mathrm{SC}$ receiver is received as a signal from the first input when this signal is greater than the signal at another input or as a signal from another input when the signal at the second input is greater than the signal at the first input. The signal probability at the output from the $\mathrm{SC}$ receiver is calculated using the formula:

$$
\begin{aligned}
p_{x}(x) & =p_{x_{1}}(x) F_{x_{2}}(x)+p_{x_{2}}(x) F_{x_{1}}(x)= \\
& \times \frac{2}{\Gamma\left(m_{1}\right)}\left(\frac{m_{1}}{\Omega_{1}}\right)^{m_{1}} x^{2 m_{1}-1} e^{-\frac{m_{1}}{\Omega_{1}} x^{2}} \frac{1}{\Gamma\left(m_{2}\right)} \gamma\left(m_{2}, \frac{m_{2}}{\Omega_{2}} x^{\alpha}\right)+ \\
& +\frac{\alpha}{\Gamma\left(m_{2}\right)}\left(\frac{m_{2}}{\Omega_{2}}\right)^{m_{2}} x^{\alpha m_{2}-1} e^{-\frac{m_{2}}{\Omega_{2}} x^{\alpha}} \frac{1}{\Gamma\left(m_{1}\right)} \gamma\left(m_{1}, \frac{m_{1}}{\Omega_{1}} x^{2}\right) .
\end{aligned}
$$

The cumulative probability at the output from the SC receivers with two inputs is obtained as a product of cumulative probability from the first and second inputs:

$$
\begin{aligned}
F_{x}(x) & =F_{x_{1}}(x) F_{x_{2}}(x)= \\
& =\frac{1}{\Gamma\left(m_{1}\right)} \gamma\left(m_{1}, \frac{m_{1}}{\Omega_{1}} x^{2}\right) \frac{1}{\Gamma\left(m_{2}\right)} \gamma\left(m_{2}, \frac{m_{2}}{\Omega_{2}} x^{\alpha}\right) .
\end{aligned}
$$

where $\gamma(n, x)$ is an incomplete Gama function, $n$ is the order of Gama function, and $x$ is the argument (Gradshteyn \& Ryzhik, 2000).

Moments are important features of the telecommunication random signals. The $n$-th moment of the signal at the output from the $\mathrm{SC}$ receiver can be calculated as:

$$
\begin{aligned}
m_{n x} & =\overline{x^{n}}=\int_{0}^{\infty} d x x^{n} p_{x}(x)=\frac{2}{\Gamma\left(m_{1}\right)}\left(\frac{m_{1}}{\Omega_{1}}\right)^{m_{1}} x^{2 m_{1}+n-1} \\
& \times e^{-\frac{m_{1}}{\Omega_{1}} x^{2}} \frac{1}{\Gamma\left(m_{2}\right)} \gamma\left(m_{2}, \frac{m_{2}}{\Omega_{2}} x^{\alpha}\right)+\frac{\alpha}{\Gamma\left(m_{2}\right)}\left(\frac{m_{2}}{\Omega_{2}}\right)^{m_{2}} \\
& \times x^{\alpha m_{2}+n-1} e^{-\frac{m_{2}}{\Omega_{2}} x^{\alpha}} \frac{1}{\Gamma\left(m_{1}\right)} \gamma\left(m_{1}, \frac{m_{1}}{\Omega_{1}} x^{2}\right) .
\end{aligned}
$$

The joint probability density function at the output from the $\mathrm{SC}$ receiver and its first derivative is:

$$
\begin{aligned}
p_{x \dot{x}}(x \dot{x}) & =p_{x_{1} \dot{x}_{1}}(x \dot{x}) F_{x_{2}}(x)+p_{x_{2} \dot{x}_{2}}(x \dot{x}) F_{x_{1}}(x)= \\
& =\frac{2}{\Gamma\left(m_{1}\right)}\left(\frac{m_{1}}{\Omega_{1}}\right)^{m_{1}} x^{2 m_{1}-1} e^{-\frac{m_{1}}{\Omega_{1}} x^{2}} \frac{1}{\sqrt{2 \pi} \sigma_{\dot{x}_{1}}} e^{-\frac{\dot{x}^{2}}{2 \sigma_{\dot{x}_{1}}^{2}}} \frac{1}{\Gamma\left(m_{2}\right)} \\
& \times \gamma\left(m_{2}, \frac{m_{2}}{\Omega_{2}} x^{\alpha}\right)+\frac{\alpha^{2}}{2 \Gamma\left(m_{2}\right)}\left(\frac{m_{2}}{\Omega_{2}}\right)^{m_{2}} x^{\alpha m_{2}+\alpha / 2+2} e^{-\frac{m_{2}}{\Omega_{2}} x^{\alpha}} \\
& \times \frac{1}{\sqrt{2 \pi} \sigma_{\dot{x}_{2}}} e^{-\frac{\alpha^{2}}{4 \sigma_{\dot{x}_{1}}^{2}} x^{\alpha-2} \dot{x}^{2}} \frac{1}{\Gamma\left(m_{1}\right)} \gamma\left(m_{1}, \frac{m_{1}}{\Omega_{1}} x^{2}\right) .
\end{aligned}
$$

The previous expression can be used to determine the average level crossing rate (aLCR).

The aLCR of the signal at the output of the SC receiver is calculated as the mean of the first derivative of the output signal of the SC receiver. Averaging is done as the integration of the product of the first derivative and the joint probability. The aLCR of $x$ is:

$$
\begin{aligned}
& N_{x}=\int_{0}^{\infty} d \dot{x} \dot{x} p_{x \dot{x}}(x \dot{x})=\frac{2}{\Gamma\left(m_{1}\right)}\left(\frac{m_{1}}{\Omega_{1}}\right)^{m_{1}} x^{2 m_{1}-1} e^{-\frac{m_{1}}{\Omega_{1}} x^{2}} \\
& \times \frac{1}{\Gamma\left(m_{2}\right)} \gamma\left(m_{2}, \frac{m_{2}}{\Omega_{2}} x^{\alpha}\right) \int_{0}^{\infty} d \dot{x} \dot{x} \frac{1}{\sqrt{2 \pi} \sigma_{\dot{x}_{1}}} e^{-\frac{\dot{x}^{2}}{2 \sigma_{\dot{x}_{1}}^{2}}} \\
& +\frac{\alpha^{2}}{2 \Gamma\left(m_{2}\right)}\left(\frac{m_{2}}{\Omega_{2}}\right)^{m_{2}} x^{\alpha m_{2}+\alpha / 2-2} e^{-\frac{m_{2}}{\Omega_{2}} x^{\alpha}} \frac{1}{\Gamma\left(m_{1}\right)} \\
& \times \gamma\left(m_{1}, \frac{m_{1}}{\Omega_{1}} x^{2}\right) \int_{0}^{\infty} d \dot{x} \dot{x} \frac{1}{\sqrt{2 \pi} \sigma_{\dot{x}_{2}}} e^{-\frac{\alpha^{2}}{4 \sigma_{x_{1}}^{2}} x^{\alpha-2} \dot{x}^{2}}= \\
& =\frac{2}{\Gamma\left(m_{1}\right)}\left(\frac{m_{1}}{\Omega_{1}}\right)^{m_{1}} x^{2 m_{1}-1} e^{-\frac{m_{1}}{\Omega_{1}} x^{2}} \frac{1}{\Gamma\left(m_{2}\right)} \frac{1}{\sqrt{2 \pi}} \sigma_{\dot{x}_{1}} \\
& \times \gamma\left(m_{2}, \frac{m_{2}}{\Omega_{2}} x^{\alpha}\right)+\frac{\alpha^{2}}{2 \Gamma\left(m_{2}\right)}\left(\frac{m_{2}}{\Omega_{2}}\right)^{m_{2}} x^{\alpha m_{2}+\alpha / 2-2} \\
& \times e^{-\frac{m_{2}}{\Omega_{2}} x^{\alpha}} \frac{1}{\Gamma\left(m_{1}\right)} \gamma\left(m_{1}, \frac{m_{1}}{\Omega_{1}} x^{2}\right) \frac{1}{\sqrt{2 \pi}} \frac{2 \sigma_{\dot{x}_{2}}}{\alpha^{2} x^{\alpha-2}} .
\end{aligned}
$$

\section{NUMERICAL RESULTS}

The numerical results are obtained, on the basis of expression (27), and are graphically shown in Figure 3. Moreover, analytical results are confirmed by MATLAB 
simulations. The signal probability density function at the output from the SC receiver with two inputs depending on the signal envelope is shown with the constant coefficient of non-linearity of the parameter $\alpha$, and for different values of the number of the cluster $\mu$, the Nakagami- $m$ fading severity $m$ and the mean values of the signal $\Omega_{1}$ and $\Omega_{2}$. The increase of the severity fading parameter $m$ and the number of clusters $\mu$ results in narrowing the range of the function at the reception, as well as increasing the maximum value achieved for higher values of the received signal level. Increasing the value of the mean signal power $\Omega_{1}$ and $\Omega_{2}$ results in the expansion of function range of the signal probability function, as well as the reduction of the maximum value, which also confirms the simulation.

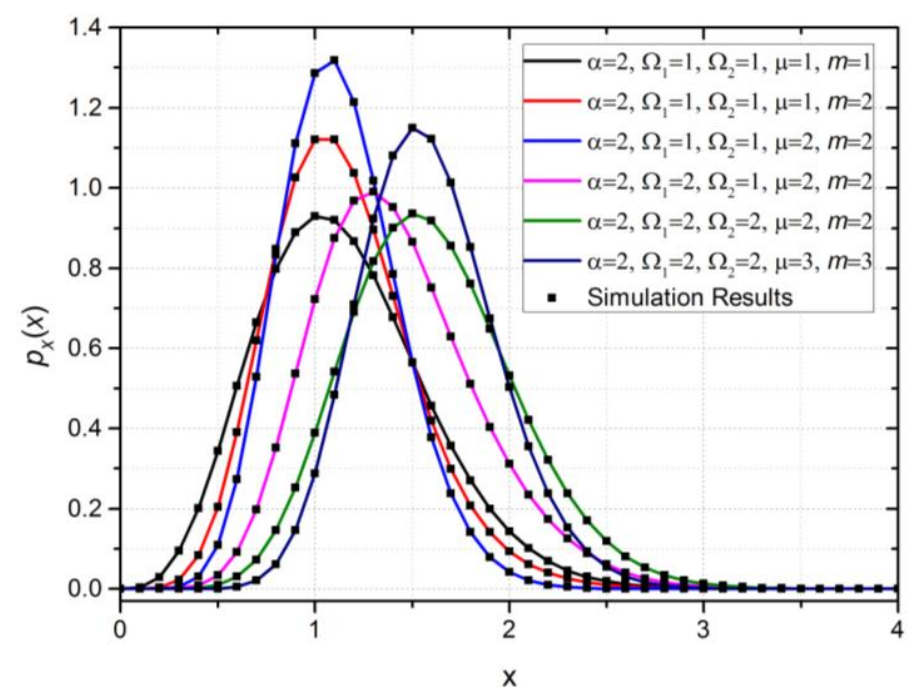

Figure 3. Graphical representation of PDF at the output from the $\mathrm{SC}$ receivers with two inputs versus the signal envelope, for different number of cluster $\mu$, the fading severity $m$ and the mean powers of the signals $\Omega_{1}$ and $\Omega_{2}$.

The numerical and simulation results for CDF are obtained, and based on expression (28), are graphically plotted versus the envelope signal in Figure 4. The cumulative probability of the signal at the output from the SC receiver with two inputs is shown with the same non-linearity coefficient parameter $\alpha$, and for different values of the number of clusters $\mu$, Nakagami- $m$ fading severity $m$ and mean powers of signal $\Omega_{1}$ and $\Omega_{2}$. Based on analytical expressions, numerical and computer simulation of the obtained results, it can be concluded that with the increase in the amplitude of the signal $x$, the cumulative probability tends to one. The growth of the cumulative probability is more dominant for the higher values of the parameters, $\mu$ and $m$. Furthermore, with the increase in the mean power values of the signals $\Omega_{1}$ and $\Omega_{2}$, CDF decreases.

Using the expression (31), in Figure 5. the normalized aLCRis graphically depicted, versus the normalized envelope of the signal $x$ and the square root of the mean power of the signal $\Omega$, where $\Omega=\Omega_{1}=\Omega_{2}$. The figure is presented with the constant values of the fading severity parameter $m$, and for different values of the nonlinearity parameter $\alpha$ as well as for different values of the clusters $\mu$. The maximum of the aLCR is higher for the higher values of the clusters $\mu$. Moreover, with the increase in $\alpha$ and $\mu$, the aLCR decreases faster, since less variation of the output signal can be observed.

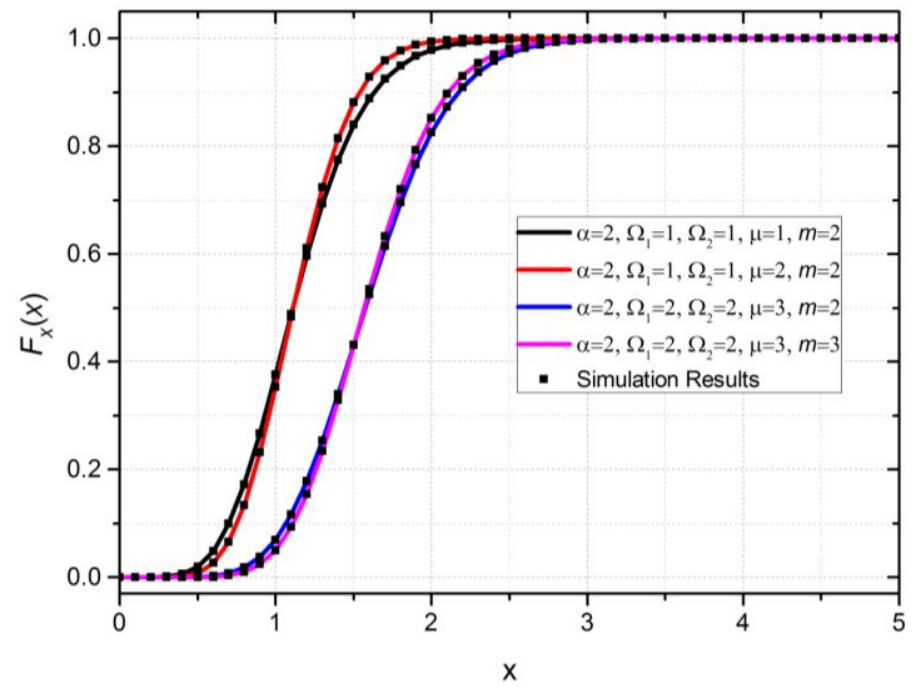

Figure 4. Graphical representation of the numerical results of the $\mathrm{CDF}$ signals at the output from the $\mathrm{SC}$ receiver with two inputs versus signal envelope, for different cluster values $\mu$, fading severity $m$ and with different mean signal powers $\Omega_{1}$ and $\Omega_{2}$.

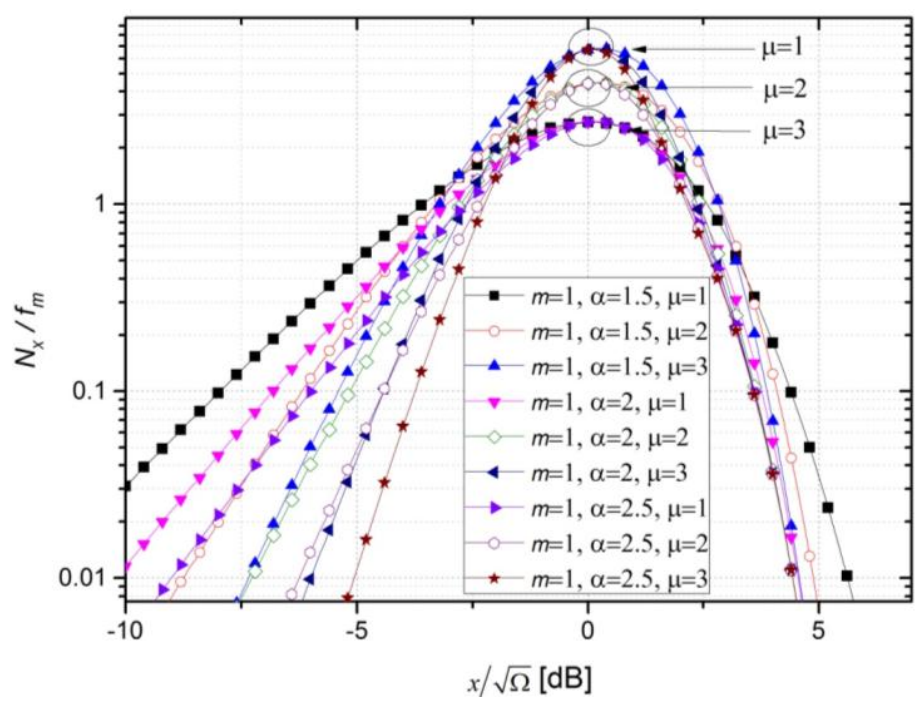

Figure 5. Normalized average number of crossing rates of the signal at the output from the SC receiver with two inputs versus normalized envelope signal, for the different values of the nonlinear coefficient $\alpha$ and the number of clusters $\mu$, and for the constant $m$

In Figure 6. the normalized aLCR is shown graphically, versus the envelope of the signal xi normalized by the square root of the signal power $\Omega$, where $\Omega=\Omega_{1}=\Omega_{2}$, with equal values of the clusters $\mu$, and for the different values of the nonlinearity parameter $\alpha$ as well as with different values of fading severity 
parameter $m$. The maximum of the aLCR is more likely to be higher for the higher $m$. With the rise in the parameter $\alpha$ and $m$, the aLCR decreases faster, since less variation of the signal can be noticed.

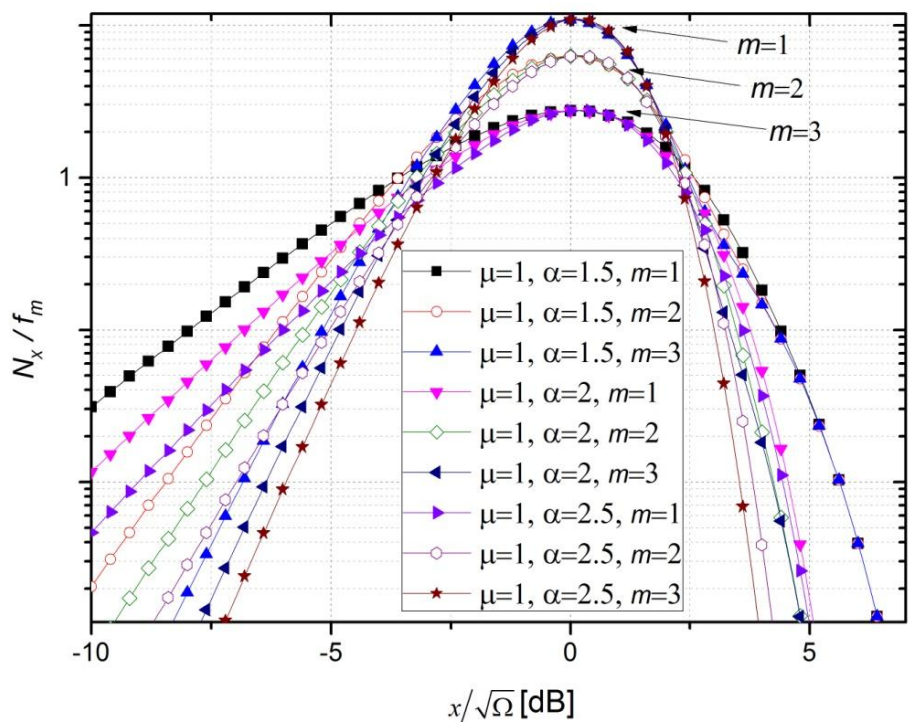

Figure 6. Normalized aLCR of the signal at the output from the SC receiver with two inputs versusthe envelope signal, for different values of the fading severity parameter $m$ and the nonlinearity coefficient $\alpha$.

\section{CONCLUSION}

This paper considers first and second order performance measures of dissimilar dual-branch SC reception in the presence of Nakagami- $m$ and $\alpha-\mu$ multipath fading at their asymmetric inputs. The closed form expressions for PDF, CDF, moments, JPDF and aLCR are derived and numerically presented for various values of system parameters. Moreover, the obtained results are verified by MATLAB simulations. It can be concluded, that for higher values of non-linearity parameter $\alpha$, number of clusters $\mu$ as well as fading severity parameter $m$, the system performances in general can be improved. Our future is going to consider performance measures of dissimilar multibranch SC reception.

\section{ACKNOWLEDGMENTS}

The work described in this paper was supported by the grant from the Serbian Ministry of Education and Science (Project no. III 44006).

\section{REFERENCES}

Analog Devices, "GaAs MMIC SPDT Non-Reflective Switch, DC-12 GHz", HMC232ALP4E datasheet, 2015 [Revised: Aug. 2018]
Bithas, P. S., Efthymoglou, G. P., \& Kanatas, A. G. 2016. A cooperative relay selection scheme in V2V communications under interference and outdated CSI. In 2016 IEEE 27th Annual International Symposium on Personal, Indoor, and Mobile Radio Communications (PIMRC). Institute of Electrical and Electronics Engineers (IEEE)., pp. 1-6. doi:10.1109/pimrc.2016.7794741

Djosic, D. B., Stefanovic, D. M., \& Stefanovic, C. M. 2016. Level Crossing Rate of Macro-diversity System with Two Micro-diversity SC Receivers over Correlated Gamma Shadowed $\alpha-\mu$ Multipath Fading Channels. IETE Journal of Research, 62(2), pp. 140-145. doi:10.1080/03772063.2015.1075913

Gradshteyn, I. S., \& Ryzhik, I. M. 2000. Table of Integrals, Series, and Products. New York: Academic. 6th ed.

Laiyemo, A. O., Pennanen, H., Pirinen, P., \& Latva-aho, M. 2017. Transmission Strategies for Throughput Maximization in High-Speed-Train Communications: From Theoretical Study to Practical Algorithms. IEEE Transactions on Vehicular Technology, 66(4), pp. 2997-3011. doi:10.1109/tvt.2016.2586118

Middleton, D. 1960. An introduction to statistical communication theory. New York: McGraw-Hill.

Milic, D., Djosic, D., Stefanovic, C., Panic, S., \& Stefanovic, M. 2015. Second order statistics of the SC receiver over Rician fading channels in the presence of multiple Nakagami- $\mathrm{m}$ interferers. International Journal of Numerical Modelling: Electronic Networks, Devices and Fields, 29(2), pp. 222-229. doi:10.1002/jnm.2065

Nakagami, M. 1960. The m-Distribution-A General Formula of Intensity Distribution of Rapid Fading. In Statistical Methods in Radio Wave Propagation.Elsevier BV., pp. 3-36. doi:10.1016/b978-0-08-009306-2.50005-4

Panajotović, A., Sekulović, N., Drača, D., Stefanović, M., \& Stefanović, Č. 2013. Average Fade Duration of Dual Selection Diversity over Correlated Unbalanced Nakagami-m Fading Channels in the Presence of Cochannel Interference. Frequenz, 67(11-12). doi:10.1515/freq-20130005

Panic, S., Stefanovic, C., \& Milosevic, H. 2017. SIR Based Performance Analysis of Dual-Branch SC Over Correlated kappa-mu Fading Channels. In E. Sabir, A. A. García, M. Ghogho, \& M. Debbah Eds., Cham: Springer Science and Business Media LLC., pp. 542-549. doi:10.1007/978-3-31968179-5_47

Simon, M. K., \& Alouini, M. 2000. Digital Communication Over Fading Channels. New York, USA: Wiley. doi:10.1002/0471200697

Stefanović, Č., Pratesi, M., \& Santucci, F. 2018. Performance evaluation of cooperative communications over fading channels in vehicular networks. In 2nd URSI Atlantic Science Radio Meeting (AT-RASC).

Stefanovic, C., Panic, S., Jovkovic, S., Simonovic, M., \& Stefanovic, M. 2017. Outage Probability of SIR Based SC Macro-Diversity Reception in Gamma Shadowed Rayleigh Multipath Fading Environment. In 2017 Sensor Signal Processing for Defence Conference (SSPD). Institute of Electrical and Electronics Engineers (IEEE)., pp. 1-5. doi:10.1109/sspd.2017.8233255 
Stefanovic, C. M. 2017. LCR of amplify and forward wireless relay systems in general alpha-mu fading environment. In 2017 25th Telecommunications Forum (TELFOR). Institute of Electrical and Electronics Engineers (IEEE), pp. 1-6. doi:10.1109/telfor.2017.8249344

Stefanović, Č., \& Đošić, D. 2016. The LCR of wireless macrodiversity SSC receiver in the presence of gamma shadowed kappa-mu fading. The University Thought Publication in Natural Sciences, 6(2), pp. 32-37. doi:10.5937/univtho6-12527

Stefanović, Č., Jakšić, B., Spalević, P., Panić, S., \& Trajčevski, Z. 2013. Performance analysis of selection combining over correlated Nakagami-m fading channels with constant correlation model for desired signal and cochannel interference. Radioengineering, 22(4), pp. 1176-1181.
R, S., Roy, R., \& Selvaraj, M. D. 2016. Performance Comparison of Selection Combining With Full CSI and Switch-andExamine Combining With and Without Post-Selection. IEEE Transactions on Vehicular Technology, 65(5), pp. 32173230. doi:10.1109/tvt.2015.2434836

Yacoub, M. D. 2007. The alpha-mu Distribution: A Physical Fading Model for the Stacy Distribution. IEEE Transactions on Vehicular Technology, 56(1), pp. 27-34. doi:10.1109/tvt.2006.883753

Yilmaz, A., \& Kucur, O. 2014. Performances of Transmit Antenna Selection, Receive Antenna Selection, and Maximal-Ratio-Combining-Based Hybrid Techniques in the Presence of Feedback Errors. IEEE Transactions on Vehicular Technology, 63(4), pp. 1976-1982. doi:10.1109/tvt.2013.2267962 